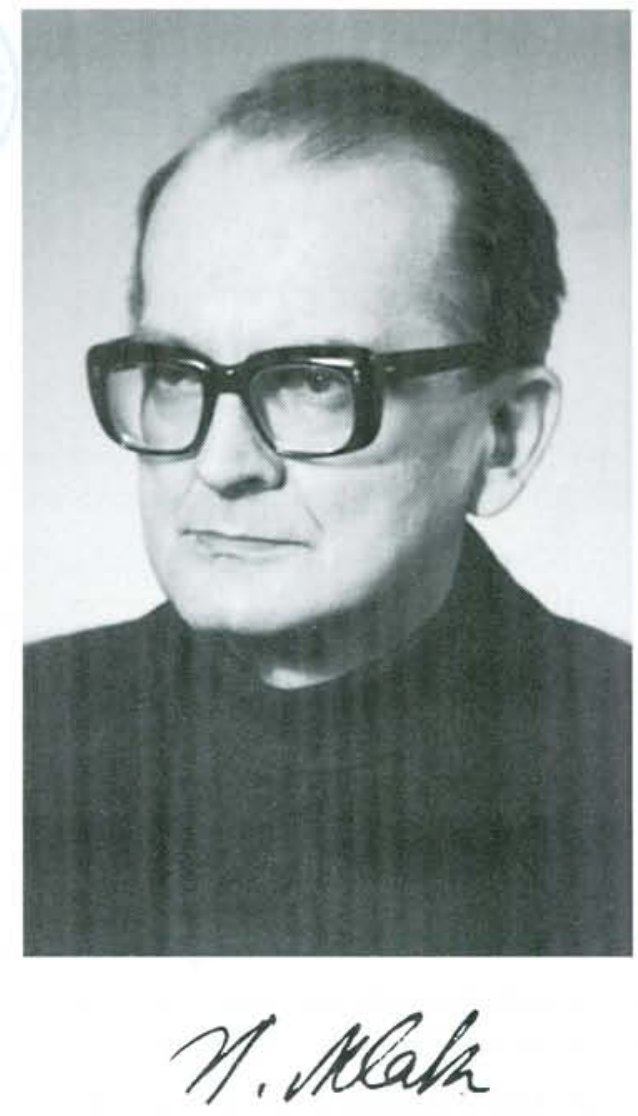




\title{
Włodzimierz Mlak (1931-1994)
}

\author{
by J. Janas (Kraków), Cz. Olech (Warszawa) \\ and F. H. Szafraniec (Kraków)
}

Włodzimierz Mlak was born on June 9, 1931 in Sosnowiec where his father was a teacher. After finishing A. Witkowski Lyceum in Cracow in 1949 he entered the Jagiellonian University as a student of mathematics. He graduated from the University in 1954. In 1958 he received his doctor degree at the Jagiellonian University under the supervision of Professor Jacek Szarski. From 1954 till 1961 he worked at the Jagiellonian University and at the same time he held a part time position at the Institute of Mathematics of the Polish Academy of Sciences; in 1961 he chose to work in the Academy for the rest of his outstanding scientific career. On April 10, 1994 Włodzimierz Mlak passed away.

The research interest of Włodzimierz Mlak at the early stage of his activity was highly influenced by the Seminar of Tadeusz Ważewski and Jacek Szarski. The main topic of the seminar at that time was the qualitative theory of differential equations and in particular differential inequalities and their applications. Mlak soon became an active member of this seminar. His papers [1]-[27] reflect this influence. He was responsible for starting the theory of differential inequalities in infinite-dimensional spaces and thus extended considerably the area of applicability of this theory developed by Ważewski and his students.

Tadeusz Ważewski observed that it is enough to assume the differential inequality $g^{\prime}(t) \leq f(t, g(t))$ to hold if $x(t)<g(t)<x(t)+\varepsilon$, where $x^{\prime}(t)=$ $f(t, x(t)), x(0)=g(0)$ and $\varepsilon>0$, in order to conclude that $g(t)$ is bounded from above by the solution $x(t)$. He called this the epiderming effect of differential inequalities. Mlak extended this result of Ważewski to systems of $n$ ordinary differential inequalities [2] as well as to partial differential inequalities of first order [3]. He used the epidermic theorems in [10] to study the differential equation $x^{\prime}=A(t) x+f(t, x)$, where $A(t)$ is a closed linear operator defined on a linear subset of a Banach space and to obtain new existence and uniqueness theorems. Also paper [17] concerns this equation 
in a Banach space where the method of differential inequalities is applied to evaluate approximate solutions.

Stability questions are the ones where differential inequalities supply a natural and powerful method of research. Paper [4] concerns the stability problem for parabolic equations. It presents an application of a theorem on differential inequalities obtained in [5].

Mlak also extended the classical Chaplygin method to abstract differential inequalities (see [15] and also [13]).

Every system of two or more differential inequalities

$$
g_{i}^{\prime}(t) \leq f_{i}\left(t, g_{1}(t), \ldots, g_{n}(t)\right), \quad i=1, \ldots, n,
$$

implies certain inequalities between the coordinates of $g(t)$ and the solution $x(t)$ of the corresponding system of differential equations provided $f$ has certain monotonicity properties. Paper [26] gives an analog of this for infinite systems of differential inequalities.

Paper [19] written in Polish deserves a mention since it is the first attempt to apply the famous retract method of Ważewski to boundary value problems.

Most of these interesting and important results of Mlak are summarized in a well-ordered exposition included as a chapter in Jacek Szarski's monograph Differential Inequalities published in 1965, the first book on the subject in the world literature (see [33]). Włodzimierz Mlak also contributed to other parts of this book thus making it more complete and valuable.

Having become professor he started the Seminar on Hilbert Space Operators introducing successfully this new field in Cracow. The Seminar attracted a group of young mathematicians who still work in operator theory.

Mlak spent the academic year 1961/62 at the University of Maryland as a visiting professor, teaching differential equations and inequalities. During that stay he turned his interests to the theory of Hilbert space operators. At that time one of the most intensively developing fields of this theory was focused on the concept of dilation of operators in Hilbert spaces. Mlak's first paper [28] in this area gave an interesting and useful characterization of completely non-unitary contractions in a Hilbert space.

This result can be briefly described as follows. Let $T$ be a completely non-unitary contraction in a Hilbert space $H$. Suppose that $E$ is the spectral measure of a minimal unitary dilation $V$ of $T$. Then the scalar measure $\mu_{f}$ defined by $\mu_{f}(\Delta)=(E(\Delta) f, f), 0 \neq f \in H, \Delta \subset \Gamma$, is absolutely continuous with respect to the Lebesgue measure $m$ on the unit circle $\Gamma$, and

$$
\log \frac{d \mu_{f}}{d m} \in L^{1}(m)
$$

Since then dilation theory became a favourite topic of Mlak's interests and resulted in a series of papers [29]-[31], [34]-[36], [37], [39], [44], [53]- 
[56], [63], [64]. These papers were quoted on different occasions by many authors, in particular in the classical monograph of Sz.-Nagy and C. Foiaş $[\mathrm{N}-\mathrm{F}]$. Mlak's contribution to dilation theory was not only in proving new theorems but also in polishing and synthesizing results of others in this area. Mlak was the first who brought together scattered results in dilation theory and published the original monographic work [32], before the book $[\mathrm{N}-\mathrm{F}]$ of Sz.-Nagy and C. Foiaş has appeared. Much later, another monographic work [60] in the same area was completed. It was at that time that dilation theory came of age. These two monographs arose as a result of Mlak's rich experience and deep thoughts on the subject. He always wished to share his knowledge with others; this was the dominating feature of his personality.

Mlak often used as a tool semi-spectral integrals, i.e. integrals with respect to semi-spectral measures. His penetrating study of the generalized von Neumann inequality gave him a new idea of semi-spectral integrals of operator-valued functions commuting with such measures. This tool allowed him to find far reaching generalizations of the von Neumann inequality [48], [51], now known as the Arveson-Mlak-Parrott inequality (cf. [FN]).

The dilation-theoretic aspect of his thinking was also successfully carried over to the analysis of stochastic processes. As an example of this approach, paper [62] dedicated to the memory of J. Szarski should be mentioned. In this paper Mlak developed the theory of conditionally positive definite (c.p.d.) functions on linear metric spaces. Let $\mathcal{E}$ be a complex linear space and let $X$ be an abstract set. Suppose we are given a family $b=\left\{b_{x, y}(f, g)\right\}_{x, y \in X}$ of bilinear forms in $f, g \in \mathcal{E}$. Recall that $b$ is conditionally positive definite if $\sum_{j, k=1}^{n} b_{x_{j}, x_{k}}\left(f_{j}, f_{k}\right) \geq 0$ for every $n=1,2, \ldots$ and all $x_{1}, \ldots, x_{n} \in X$ and $f_{1}, \ldots, f_{n} \in \mathcal{E}$ such that $\sum_{j=1}^{n} f_{j}=0$. Mlak mostly studied c.p.d. functions of the form $b_{x-y}\{f, g\}$ in the case of $X$ being a real separable Hilbert space. He found the unique representation of the so-called structural $d$ of $b$ in terms of Lévy-Khinchin's formula for $b$ (due to Varadhan). This result was known before as the Yaglom formula only in the case $X=\mathbb{R}^{n}$.

After publication of Parrott's counterexample Mlak realised that dilation methods are not universal and he turned to function algebra techniques. This point of view is clearly represented in a number of papers (see [45], [46], [49], [52], [61]). In order to describe results contained in these works, first recall that for a compact Hausdorff space $X$ by a function algebra $A \subset C(X)$ we mean a Banach algebra which contains constants and separates the points of $X$. Let $T: A \rightarrow L(H)$ be a contractive unital homomorphism of $A$ into the algebra of all bounded linear operators in a complex Hilbert space $H$.

A typical example of Mlak's dilation-free approach to the study of the representation $T$ of $A$ is given in [45]. In this paper he applied the abstract version of the M. and F. Riesz theorem to prove some decomposition theorems of an arbitrary algebra $A \subset C(X)$ with respect to the totality 
of Gleason parts of $A$. These decompositions are especially useful in the theory of commuting contractions (see the book by Paulsen-Douglas [DP]), because they are proved in an abstract setting; an extension of this approach to non-contractive representations was proposed in $[\mathrm{Sz}]$.

In [52] Mlak found, also in a completely dilation-free way, a general result about partitions of spectral sets. Because this result has been used by several authors let us describe it more precisely. For a given compact set $X \subset \mathbb{C}$ define the function algebra $R(X)$ as the closure in $C(X)$ of the algebra of rational functions having poles off $X$. If $T: R(X) \rightarrow L(H)$ is a unital contractive representation, then we say $X$ is a spectral set for $V=T\left(u_{1}\right)$, where $u_{1}(z) \equiv z$.

Let $G_{i}(i=1,2, \ldots)$ be the sequence of all non-peak point parts for $R(X)$. The main result of [52] shows that $V=\bigoplus_{i=1}^{\infty} V_{i} \oplus V_{0}$, where $V_{i}$ has $\bar{G}_{i}$ as a spectral set for $i>0$, the representation of $R\left(\bar{G}_{i}\right)$ generated by $V_{i}$ $(i>0)$ has elementary measures absolutely continuous with respect to the set $M_{z}$ of all representing measures for $z \in G_{i}$, and $V_{0}$ is a normal operator with spectrum carried by $\partial X$ (cf. [C], Section 21, "The Lautzenheiser-Mlak theorem").

The language of operator-valued representations turned out to be fruitful in Mlak's study of commutants of subnormal operators. Recall that a bounded operator $S$ in $H$ is subnormal if there exists a larger Hilbert space $K$ and a normal operator $N$ in $K$ such that $S h=N h, h \in H$. One of the basic questions asked in the analysis of subnormal $S$ is: when does an operator $X$ commuting with $S$ extend to a bounded operator $\widetilde{X}$ in $K$ commuting with $N$ ? In [49] Mlak found an elegant sufficient condition (for a function algebra $A$ ), which guarantees an affirmative answer to that question. His condition says that the finite sums $\sum_{k}\left|u_{k}\right|^{2}, u_{k} \in A$, must be dense in the uniform norm in the cone of positive continuous functions on the Shilov boundary $\partial A$ of $A$. This approach has been further developed in $[\mathrm{J}]$ in the context of several commuting subnormal operators.

An interesting result on Szegő measures relative to $\left.R(X)\right|_{\partial X}$ is contained in [61]. Here $X$ is a compact set in the complex plane. Let $G$ be a non-peak point Gleason part of the spectrum of $R(X)$. Mlak proved that a Szego" measure $\mu$ (supported on $\partial X$ ) which is absolutely continuous with respect to a representing measure $m$ for some $\varphi \in G$ must be a Szegő measure relative to $\left.R(\bar{G})\right|_{\partial \bar{G}}$. The results of that paper have found an application in the theory of subnormal operators with infinitely connected spectrum (taken up in $[\mathrm{R}])$.

There is a group of papers which cannot be included in any of the above classes. One of them is a joint paper with C. Foiaş [35], where they introduced and proved an interesting extension of the spectral mapping theorem for the algebra $H^{\infty}$ of functions holomorphic and bounded in the unit disc $\mathbb{D}$. 
As a corollary of this result, for a completely non-unitary contraction $T$ and $u \in H^{\infty}$ which has continuous radial limits at every point in the intersection of the spectrum $\sigma(T)$ and the unit circle $\Gamma$, one has the spectral mapping theorem: $\sigma(u(T))=u(\sigma(T))$. Their result was applied by Brown, Chevreau and Pearcy in the theory of invariant subspaces $[\mathrm{BCP}]$.

A particular position in Mlak's achievements is occupied by paper [59]. In this short article he proved that any operator $S$ in $H$ with spectrum contained in $\overline{\mathbb{D}}$, for which the functions $U_{f, g}(z)=\left((I-z S)^{-1} f, g\right)$ are in the Hardy space $H^{1}$ over $\mathbb{D}$, must have spectral radius strictly less than one. Although this theorem may be regarded as a particular case of a more general approach developed at the same time by N. K. Nikol'skiı̌, Mlak's method of proof is completely different.

In turn, in a beautiful paper [69] dedicated to the memory of F. Leja he presented an original approach to composition (Koopman) operators $C_{a}: f \mapsto f \circ a$ acting in $L^{2}(\mathbb{R}, p)$, where $d p=\frac{1}{\sqrt{2 \pi}} e^{-x^{2} / 2} d x$. This partly expository paper contains results of various character: some relations between several models and interpretation of composition operators, analysis of their finite and infinite tensor products and applications to certain integral equations. The paper inspired in an essential way Mlak's student Jan Stochel [S] who continued the study of composition operators in $L^{2}\left(\mathbb{R}^{n}, d \mu\right)$.

In the last period of his scientific activity Mlak became interested in mathematical problems related to phase operators investigated in theoretical physics. As a result of his interests he began a detailed study of so-called circular operators (see [68], [73], [75]-[79]).

We say that a bounded operator $T$ is an $(A, \alpha)$-operator (or a circular operator) if

$$
e^{-i t A} T e^{i t A}=e^{i \alpha t} T
$$

for all real $t$, where $A$ is a selfadjoint operator and $\alpha \neq 0$ is a real number. Mlak proved in [68] (see also [76]) that (1) is equivalent to

$$
T e^{-t A}=e^{\alpha t} e^{-t A} T, \quad t \geq 0,
$$

provided $A$ is bounded from below. In [75] he analysed spectral properties of semi-normal solutions $T$ of (1) and found several decompositions related to the interplay between $A$ and $T$.

The subsequent paper [76] brings the form of a general solution of (1). In the special case when $A$ is an orthogonal sum $\bigoplus_{n=0}^{\infty} n P_{n}$, where $P_{n}$ are projections and $\operatorname{dim} P_{n}=d(n=0,1,2, \ldots)$, Mlak proved that $T$ must be a unilateral operator weighted shift of multiplicity $d$.

In another paper [73] of this series, dedicated to the memory of Z. Opial, Mlak found an interesting extension of the theorem (due to Ifantis) concern- 
ing the triviality of a selfadjoint solution $N$ of the equation

$$
(N F-F N) f=i f, \quad f \in M,
$$

where $F$ is a bounded selfadjoint operator and $M$ is a linear dense subspace in $H$ such that $F M \subset M \subset D(F)$. Mlak considered the more general case when $F$ is replaced by a general operator and $N$ by a normal operator with zero-dimensional spectrum.

His experience as a teacher resulted in the monographic book [47]. The book became popular and useful; several editions and translations into English [74] prove this. It gives the reader a very accessible introduction to the theory of linear operators in Hilbert spaces, containing in particular a clear presentation of integrals with respect to semi-spectral measures.

\section{W. Mlak's publications}

[1] Note on the mean value theorem, Ann. Polon. Math. 3 (1956), 29-31.

[2] On the epidermic effect for ordinary differential inequalities of the first order, ibid. 3 (1956), 37-40.

[3] The epidermic effect for partial differential inequalities of the first order, ibid. 3 (1956), 157-164.

[4] Remarks on the stability problem for parabolic equations, ibid. 3 (1957), 343-348.

[5] Differential inequalities of parabolic type, ibid. 3 (1957), 349-354.

[6] A note on non-local existence of solutions of ordinary differential equations, ibid. 4 (1958), 344-347.

[7] Differential inequalities in linear spaces, ibid. 5 (1958), 95-101.

[8] Limitations and dependence on parameter of solutions of non-stationary almost linear differential operators equations, Bull. Acad. Polon. Sci. Sér. Sci. Math. Astronom. Phys. 6 (1958), 677-682.

[9] The first boundary value problem for a non-linear parabolic equation, Ann. Polon. Math. 5 (1958), 257-262.

[9a] Limitation of solutions of parabolic equations, ibid. 5 (1958), 237-245.

[10] Limitations and dependence on parameter of solutions of non-stationary differential operator equations, ibid. 6 (1959), 305-322.

[11] Zagadnienia brzegowe dla równań parabolicznych prawie liniowych [Boundary value problems for almost linear parabolic equations], Zeszyty Nauk. Uniw. Jagielloń. Prace Mat. 5 (1959), 5-18 (in Polish).

[12] Integration of differential equations with unbounded operators in abstract $(L)$-spaces, Bull. Acad. Polon. Sci. Sér. Sci. Math. Astronom. Phys. 8 (1960), 163-168.

[13] Parabolic differential inequalities and Chaplighin's method, Ann. Polon. Math. 8 (1960), 139-153.

[14] Differential inequalities with unbounded operators in Banach spaces, ibid. 9 (1960), 101-111.

[15] Note on abstract differential inequalities and Chaplighin method, ibid. 10 (1961), 253-271.

[16] Note on abstract linear differential inequalities, Rev. Math. Pures Appl. 6 (1961), 655-657. 
[17] A note on approximation of solutions of abstract differential equations, Ann. Polon. Math. 10 (1961), 273-278.

[18] On a linear differential inequality of parabolic type, Bull. Acad. Polon. Sci. Sér. Sci. Math. Astronom. Phys. 7 (1959), 653-656.

[19] O pierwszym zagadnieniu brzegowym dla równania $y^{\prime \prime}=f\left(t, y, y^{\prime}\right)$ [On the first boundary value problem for the equation $\left.y^{\prime \prime}=f\left(t, y, y^{\prime}\right)\right]$, Zeszyty Nauk. Uniw. Jagielloń. Prace Mat. 6 (1961), 21-26 (in Polish).

[20] Monotonic sequences of approximate solutions of the equation $y^{\prime}=f(t, y)$, Mathematica (Cluj) 4 (1962), 53-56.

[21] Differential inequalities, Lecture Notes, University of Maryland, 1962, 96 pp.

[22] Estimates of solutions of hyperbolic systems of differential equations in two independent variables, Ann. Polon. Math. 12 (1962), 191-197.

[23] Note on maximal solutions of differential equations, Contributions to Differential Equations 1 (1963), 461-465.

[24] Integration of the first order partial differential inequality with distributions, Colloq. Math. 10 (1963), 147-150.

[25] Integration of linear differential inequalities with distributions, Contributions to Differential Equations 2 (1963), 265-268.

[26] Integration of infinite systems of differential inequalities (with Cz. Olech), Ann. Polon. Math. 13 (1963), 105-112.

[27] An example of the equation $u_{t}=u_{x x}+f(x, t, u)$ with distinct maximum and minimum solutions of a mixed problem, ibid. 13 (1963), 101-103.

[28] Characterization of completely non-unitary contractions in Hilbert spaces, Bull. Acad. Polon. Sci. Sér. Sci. Math. Astronom. Phys. 11 (1963), 111-113.

[29] Note on the unitary dilation of a contraction operator, ibid. 11 (1963), 463-467.

[30] Some prediction theoretical properties of unitary dilations, ibid. 12 (1964), 37-42.

[31] Representations of some algebras of generalized analytic functions, ibid. 13 (1965), $211-214$.

[32] Unitary dilations of contraction operators, Rozprawy Mat. 46 (1965).

[33] Differential inequalities in linear spaces, Chapt. XI in: J. Szarski, Differential Inequalities, Monografie Mat. 43, Warszawa, 1965. Also, $\S \S 31,32,66,67$ in that book.

[34] A Hilbert space characterization of Lebesgue decomposition of certain measures, (with T. Ando), Bull. Acad. Polon. Sci. Sér. Sci. Math. Astronom. Phys. 14 (1966), 87-89.

[35] The extended spectrum of completely non-unitary contractions and the spectral mapping theorem (with C. Foiaş), Studia Math. 26 (1966), 239-245.

[36] On semi-groups of contractions in Hilbert spaces, ibid. 26 (1966), 263-272.

[37] Unitary dilations in case of ordered groups, Ann. Polon. Math. 17 (1966), 321-328.

[38] On a mean value theorem for vector-valued functions, with applications to uniqueness theorems for "right-hand-derivative" equations (with A. K. Aziz and J. B. Diaz), J. Math. Anal. Appl. 16 (1966), 302-307.

[39] Positive definite contraction valued functions, Bull. Acad. Polon. Sci. Sér. Sci. Math. Astronom. Phys. 15 (1967), 509-512.

[40] Hyponormal contractions, Colloq. Math. 18 (1967), 137-142.

[41] A note on Szegö type properties of semi-spectral measures, Studia Math. 31 (1968), $241-251$.

[42] On the Chaplighin method for partial differential equations of the first order (with E. Schechter), Ann. Polon. Math. 22 (1969), 1-18. 
[43] Absolutely continuous operator valued representations of function algebras, Bull. Acad. Polon. Sci. Sér. Sci. Math. Astronom. Phys. 17 (1969), 547-550.

[44] Spectral properties of Q-dilations, ibid. 17 (1969), 397-400.

[45] Decompositions and extensions of operator valued representations of function algebras, Acta Sci. Math. (Szeged) 30 (1969), 181-193.

[46] Decompositions of operator-valued representations of function algebras, Studia Math. 36 (1970), 111-123.

[47] Wstẹp do teorii przestrzeni Hilberta [Introduction to the theory of Hilbert spaces], Biblioteka Mat. 35, PWN, 1970, 398 pp. (2nd ed. 1977, 4th revised ed. 1987).

[48] Note on the von Neumann inequality, Bull. Acad. Polon. Sci. Sér. Sci. Math. Astronom. Phys. 19 (1971), 843-848.

[49] Commutants of subnormal operators, ibid. 19 (1971), 837-842.

[50] Intertwining operators, Studia Math. 43 (1972), 219-233.

[51] Inequalities for semi-spectral integrals (with C. Ryll-Nardzewski), Bull. Acad. Polon. Sci. Sér. Sci. Math. Astronom. Phys. 20 (1972), 11-16.

[52] Partitions of spectral sets, Ann. Polon. Math. 25 (1972), 273-280.

[53] On convergence properties of operators of class $\mathscr{C}_{\varrho}$, Acta Sci. Math. (Szeged) 33 (1972), 353-354.

[54] Note on unitary dilations of two commuting contractions, Bull. Acad. Polon. Sci. Sér. Sci. Math. Astronom. Phys. 21 (1973), 941-944.

[55] Operator inequalities and related dilations, Acta Sci. Math. (Szeged) 34 (1973), 273-278.

[56] Semi-spectral measures of two commuting contractions, Bull. Acad. Polon. Sci. Sér. Sci. Math. Astronom. Phys. 21 (1973), 695-698.

[56a] Decompositions of polynomially bounded operators, ibid. 21 (1973), 317-322.

[56b] Positive definite operator functions which are representations (with C. Ryll-Nardzewski), ibid. 22 (1974), 1111-1116.

[57] Algebraic polynomially bounded operators, Ann. Polon. Math. 29 (1974), 133-139.

[58] Operator valued representations of function algebras, in: Linear Operators Approx. II, Proc. Conf. Oberwolfach, 1974, P. Butzer and B. Sz.-Nagy (eds.), Birkhäuser, 1974, 49-79.

[59] On a theorem of Lebow, Ann. Polon. Math. 35 (1977), 107-109.

[60] Dilations of Hilbert space operators (general theory), Dissertationes Math. 153 (1978).

[61] Szegö measures related to compact plane sets, Comment. Math. Prace Mat., Tomus Specialis in Honorem Ladislai Orlicz, I, 1978, 239-249.

[62] Dissipative random processes, in: Probability Theory on Vector Spaces, Proc. Conf. Trzebieszowice, 1977, Lecture Notes in Math. 828, Springer, Berlin, 1980, 139-169.

[63] Dilations with operator multipliers (with F. H. Szafraniec), in: Probability Theory on Vector Spaces. II, Proc., Błażejewko, 1979, A. Weron (ed.), Lecture Notes in Math. 828, Springer, Berlin, 1980, 208-214.

[64] Dilations of Banach space operator valued functions (with A. Weron), Ann. Polon. Math. 38 (1980), 295-303.

[65] A note on general dilation theorems, in: Spectral Theory, Banach Center Publ. 8, PWN, Warszawa, 1982, 347-352.

[66] Conditionally positive definite functions on linear spaces, Ann. Polon. Math. 42 (1983), 187-239.

[67] Jacek Szarski 6.II.1921-21.II.1980 (with W. Kleiner and A. Pliś), ibid. 42 (1983), $1-3$. 
[68] Notes on quantum circular operators, Part I, preprint 303, Inst. Math., Polish Acad. Sci., 1984.

[69] Operators induced by transformations of Gaussian variables, Ann. Polon. Math. 46 (1985), 197-212.

[70] Continuity properties of conditionally positive definite functions on linear spaces, Univ. Iagel. Acta Math. 25 (1985), 109-113.

[71] Note on unitary circular operators, ibid. 27 (1988), 303-307.

[72] The Schrödinger type couples related to weighted shifts, ibid. 27 (1988), 297-301.

[73] Commutation relations involving spectrally scattered operators, Ann. Polon. Math. 51 (1990), 255-258.

[73a] In memory of Professor Zdzistaw Opial, ibid. 51 (1990), 5.

[74] Hilbert Spaces and Operator Theory, Polish Sci. Publ., Warszawa, and Kluwer Academic Publ., Dordrecht, 1991.

[75] Time dependent circular operators, Univ. Iagel. Acta Math. 29 (1992), 177-180.

[76] Notes on circular operators I, ibid. 29 (1992), 145-152.

[77] Notes on circular operators II, ibid. 29 (1992), 153-162.

[78] Notes on circular operators III, ibid. 29 (1992), 163-170.

[79] Notes on circular operators IV, ibid. 29 (1992), 171-175.

[80] Quantum phase and circular operators (with M. Słociński), ibid. 29 (1992), 133-144.

[81] A note on coherent states related to weighted shifts (with J. Stochel), Publ. RIMS Kyoto Univ. 28 (1992), 587-593.

\section{Other references}

[BCP] S. Brown, B. Chevreau and C. Pearcy, Contractions with rich spectrum have invariant subspaces, J. Operator Theory 1 (1979), 123-136.

[C] J. B. Conway, The Theory of Subnormal Operators, Math. Surveys Monographs 36, Amer. Math. Soc., Providence, R.I., 1991.

[DP] R. G. Douglas and V. I. Paulsen, Hilbert Modules over Function Algebras, Longman Scientific \& Technical, N.Y., 1989.

[FN] M. Fujii and M. Nakamura, A comment on the inequalities of Arveson-Parrott and Mlak, Math. Japon. 20 (1975), special issue, 65-67.

[J] J. Janas, Some applications of functions of several complex variables to Toeplitz and subnormal operators, Ann. Polon. Math. 40 (1983), 185-192.

[R] K. Rudol, The generalised Wold Decomposition for subnormal operators, Integral Equations Oper. Theory 11 (1988), 420-436.

[S] J. S tochel, Seminormal composition operators on $L^{2}$ spaces induced by matrices, Hokkaido Math. J. 19 (1990), 307-324.

[Sz] F. H. Szafraniec, Decompositions of non-contractive operator valued representations of Banach algebras, Studia Math. 42(1972), 97-108.

[NF] B. Sz.-Nagy and C. Foiaş, Harmonic Analysis of Operators on Hilbert Space, North-Holland, Amsterdam, 1970. 